\title{
Device Modeling with Memristor Element for Chaotic System
}

\author{
Savarimuthu Prakash, Sam Thomas
}

\begin{abstract}
The proposed circuit is a basic electric circuit that produces chaos whcih consists three energy storage devices, a dissipative device, and a non-linear device. This paper investigates the use of a memristor as the nonlinear device in Chua's circuit. The memristor is represented mathematically by the port equation and the state equation. The memristor state is defined by the past and present values of input voltage rather than by a piecewise linear or cubic polynomial function. The proposed circuit shows that the circuit undergoes chaotic oscillations that are sensitive to the initial conditions.
\end{abstract}

Keywords: Memristor, Chaos, Nonlinear dynamics, Chaotic circuit, Chua's circuit

\section{INTRODUCTION}

In electronics are only three fundamental two terminal passive components, which is a disspative element (resistor) and two energy storage elements (capacitor and inductor). The devices are defined using the circuit variables (voltage,current, charge and flux). Chua [1], in 1971, mathematically postulated a fourth fundamental circuit component relating both charge and magnetic flux. The nanodevice has the property of remembring the last applied input. The memristor is an electrocic device (or a class of memristive devices) that changes its memristance based on the amount of charge flowing through it. The memristor acts as a resistor which has memory property and exhibits fascinating non-linear characteristics. Chua's circuit has an inductor and a capacitor forming a tank circuit connected in series via a resistor to another parallel combination of a capacitor and Chua's nonlinear device, as reprsented in Fig. 1. Thus, a new Chua's chaotic circuit may be constructed using three energy storage components and an active Chua's diode for generating chaotic output. Chua's circuit has applications in secure communication, chaos control, and image processing[2-4].

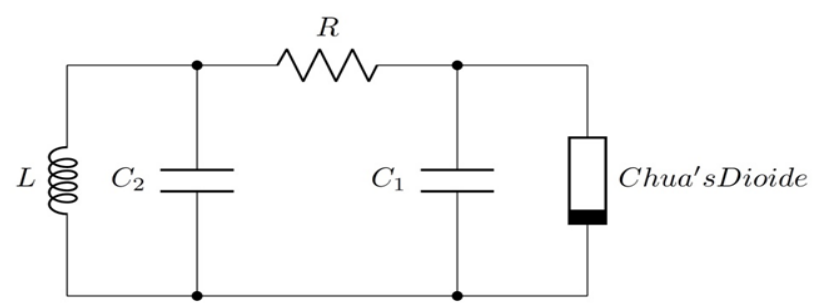

Figure 1: Chua's chaotic circuit.

Revised Manuscript Received on August 14, 2019.

Savarimuthu Prakash, Department of Electronics and Communication Engineering, SSE, SIMATS, Chennai, Tamil Nadu, India.(email: aprakashs.sse@saveetha.com)

Sam Thomas, Faculty of Information and Communication, Anna University, Kotturpuram, Chennai, Tamil Nadu, India

\section{LINEAR DRIFT MEMRISTOR AND CHUA'S CIRCUIT}

The linear drift memristor model put forth by HP has $\mathrm{TiO} 2$ and $\mathrm{TiO} 2-\mathrm{X}$ positioned between platinum electrodes (Figure 2). A voltage with a positive bias applied at the pt electrode on the $\mathrm{TiO} 2$ side repels all of the oxygen vacancies which are positiviely charged. Thus, the doped region $(\mathrm{TiO} 2-\mathrm{X})$ widens ( $\mathrm{w}$ increases) and the conductivity increases (device resistance decreases). When a voltage with negative bias is applied at the same terminal attracts oxygen vacancies which are the positively charged, reducing the $\mathrm{TiO} 2-\mathrm{X}$ region (w decreases) and the conductivity (device resistance increases). As the $\mathrm{TiO} 2-\mathrm{X}$ region grows, the width of the $\mathrm{TiO} 2-\mathrm{X}$ region (w) approaches the thickness (D) of the entire device, the resistance reduces, and the device is considered to be in the ON state. As w increases to the value $\mathrm{D}$, the resistance of the component will be RON which is the resistance of the doped (TiO2-X) region. As the doped region shrinks (or the $\mathrm{TiO} 2$ region expands) and the width of the undoped region approaches the thickness D of the entire device, the resistance increases, and the device is considered to be in the OFF state. ROFF is the value of the $\mathrm{TiO} 2$ region when $\mathrm{w}=0$ [5].

The memristance of the device is defined by using a series combination with a slider combination [6] in between the resistance of the $\mathrm{TiO} 2-\mathrm{X}$ region ( $\mathrm{RON}$ ) and the resistance of the $\mathrm{TiO} 2$ region (ROFF), as given by the port equation:

$$
v(t)=\left[\frac{R_{\mathrm{ON}}^{*} w(t)}{D}+R_{\mathrm{OFF}}\left(1-\frac{w(t)}{D}\right)\right] i(t)
$$

The values of both doped region resistance and undoped region resistance are controlled via the ratio w/D The rate of change of $w$ is proportional to the current $i(t)$, as given by the state equation

$$
\frac{d w}{d t}=\mu_{V} \frac{R_{\mathrm{ON}}}{D} i(t) .
$$

where dopant mobility is represented as $\mu_{V}$.

Memristors exhibit pinched hysteresis. For a sinusoidal input, the current-voltage characteristics of the device is shown in Figure. 3. As the input frequency increases, the

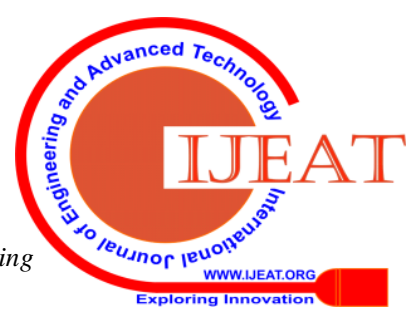


lobes in the first quadrant and third quadrant reduces to a linear form (Figure 3). Thus, at higher frequencies, the memristance property is lost and the device acts as a linear resistor. Figure 3 shows that $\mathrm{v}(\mathrm{t})$ and $\mathrm{i}(\mathrm{t})$ reach zero simultaneously, which is consistent with Eq. (1). To simulate memristor characteristics, we used OrCAD Capture and PSpice Lite 16.6. The memristance of a device ranges between positive values RON and ROFF. If Chua's diode is substituted with a memristor, the nonlinear element should have a positive slope. Because the slope of the nonlinear element characterizes the stability of an equilibrium point at the point under consideration, the memristor with a positive slope produces a stable equilibrium point. At a stable equilibrium point, all waves settle onto the equilibrium point. Therefore, no oscillations are produced. Thus, the memristor cannot be directly substituted as the nonlinear element of the Chua's circuit. For the equilibrium point to be unstable, the slope of the nonlinear element should be negative. Adding a negative resistance in series does not yield a negative slope because of the current-voltage characteristics of a memristor in series with a negative resistor yield a positive slope. A negative slope occurs for a memristor in parallel with a negative resistor.

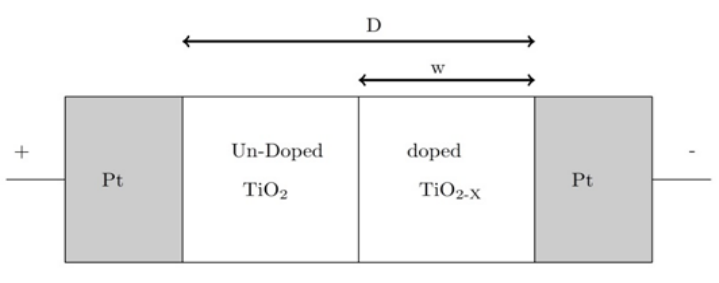

Figure 2: Memristor model of the two terminal $\mathrm{Pt} / \mathrm{TiO} / \mathrm{TiO2}-\mathrm{X} / \mathrm{Pt}$ memristor

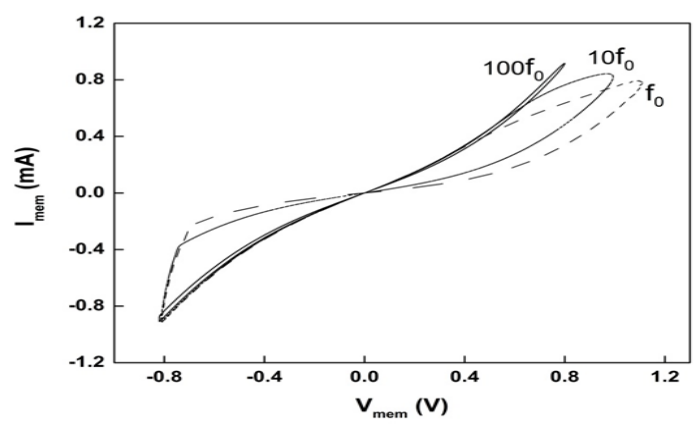

Figure 3: Hysteresis loop and Time waveforms of voltage and current: As the sweep frequency is increased ten times (10fO) and hundred times (100fO), there is a decrease in the lobes of the pinched hysteresis.

\section{PROPOSED NEW MEMRISTOR BASED CHAOTIC CIRCUIT}

Figure 4 shows chaotic circuit with the Chua's nonlinear device[7,8] substituted by a negative resistance and the memristor. The inductor $\mathrm{L}$ is in parallel with capacitor $\mathrm{C} 2$ to form a tank circuit that produces lossless oscillations. The nonlinear element locally passive, all trajectories would approach a stable equilibrium and the circuit would produce no oscillations. However, because the nonlinear element is locally active, it supplies power to the external circuit. The resistor $\mathrm{R}$ interacts with capacitor $\mathrm{C} 1$ and the nonlinear element. Because $\mathrm{R}$ is a dissipative element, it restrains the growth of the trajectory, which forms a chaotic trajectory that is constrained to a particular region (basin of attraction).

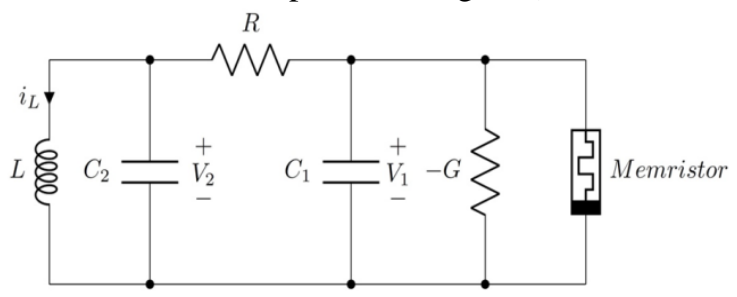

Figure 4: Proposed chaotic circuit. Chua's nonlinear element is exchanged by the parallel combination of the memristor and a negative resistance component.

Applying Kirchhoff's law to proposed chaotic circuit, the set of differential equations are obtained (Equation 3).

$$
\begin{gathered}
\frac{d v_{C 1}}{d t}=\frac{1}{C_{1}}\left[\frac{v_{C 2}-v_{C 1}}{R}+\frac{v_{C 1}}{G}-\frac{v_{C 1}}{R_{\text {mem }}(w)}\right], \\
\frac{d v_{C 2}}{d t}=\frac{1}{C_{2}}\left[\frac{v_{C 1}-v_{C 2}}{R}+i_{L}\right], \\
\frac{d i_{L}}{d t}=-\frac{v_{C 2}}{L}, \\
\frac{d w}{d t}=\mu_{V} \frac{R_{O N}}{D} v_{C 1},
\end{gathered}
$$

where and are the voltages across the capacitor $\mathrm{C} 1$ and $\mathrm{C} 2$ and is the current flowing through the inductor L, and Rmem(w) is the memristance of the memristor. The evolution of $\mathrm{w}$ is controlled by the change in (3). The nonlinear device is thus a voltage-controlled memristor.

$$
R_{\mathrm{mem}}(w)=R_{\mathrm{ON}} \frac{w(t)}{D}+R_{\mathrm{OFF}}\left(1-\frac{w(t)}{D}\right)
$$

The parameter values for the HP memristor are $\mathrm{RON}=$ $100 \Omega, \mathrm{ROFF}=10 \mathrm{k} \Omega, \mu \mathrm{V}=10-14 \mathrm{~m} 2 \mathrm{~s}-1 \mathrm{~V}-1, \mathrm{D}=10$

$\mathrm{nm}$. The circuit elements are $\mathrm{C} 1=7.5 \mathrm{nF}, \mathrm{C} 2=68 \mathrm{nF}, \mathrm{L}$ $=18 \mathrm{mH}$, and $\mathrm{R}=1.8 \mathrm{k} \Omega$.

Numerical computational methods are utilized to determine the solutions of the physical system. The RungeKutta analysis method is applied to find the solution of the differential equations of the Chua's circuit [8] with a step size of $\Delta t=0.001$. We used the ode45 function in MATLAB R2016a to solve the differential equation and analyze the solution. For the numerical analyses, the component values listed previously was applied. The resistance value $\mathrm{R}$ was varied to alter the behaviour of the proposed system. 


\section{RESULTS OF THE PROPOSED NEW MEMRISTOR BASED CHAOTIC CIRCUIT}

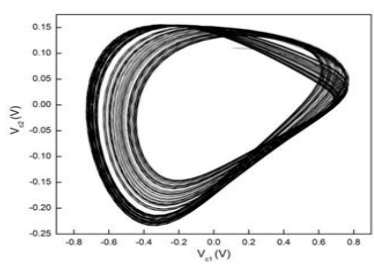

(a)

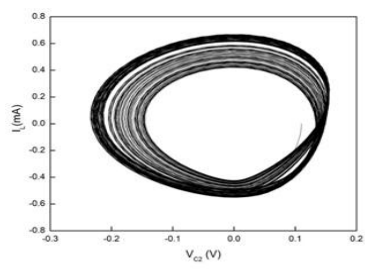

(b)
Figure 5: Chaotic trajectory and power spectrum: (a) Chaotic trajectory projection on plane; (b) Chaotic trajectory projection on plane

Figure 5 shows the chaotic trajectory and power spectrum of the circuit. The chaotic trajectory reveals a stable steadystate solution of Chua's chaotic circuit and a homoclinic orbit: this is a strange attractor. The trajectory starts from an initial condition and then moves continually around the fixed point without ever intersecting its orbit. Although the system is based on a fourth-order differential equation, for any finite-time observations, the trajectory is irregular and does not return to its starting point. The power spectrum is characteristic of broadband noise, which indicates the existence of an aperiodic waveform. The power is not concentrated in a small number of frequencies but is distributed over a wide range of frequencies. For a periodic signal, for example, the power would not be distributed but concentrated in the dc component, the fundamental frequency component, and the harmonics.

\section{CONCLUSION}

This work presents a new chaotic circuit using memristor. In which the conventional Chua's diode is replaced with a negative resistor which is in parallel with a memristor in the proposed chaotic circuit. The implemented memristor is based on the physical equations proposed by HewlettPackard. The memristor-based Chua's circuit becomes chaotic with actual values and without requiring scaling. In addition, the memristor state equation is based on past and present values of $\mathrm{w}$ and the input voltage (or current), so Chua's nonlinear element may be effortlessly replaced by a physical component of the memristor. Also, these type of chaotic behaviour system is useful in transmitting secure image and documents over wireless communication.

\section{REFERENCES}

1. L.Chua, Memristor-The missing Circuit Element. IEEE Transactions on Circuit Theory; 1971, 18, p507, .

2. B. Bao, F. Hu, M. Chen, Q. Xu, and Y. Yu, Coexistence of Multiple Attractors in an Active Diode Pair Based, International Journal of Bifurcation and Chaos; 2015, 25, 1550075.

3. G. Gandhi, G. Cserey, J. Zbrozek, and T. Roska, Anyone can build chua's circuit: Hands-on-experience with chaos theory for high school students., International Journal of Bifurcation and Chaos; 2009,19, p1113.

4. Y.V. Pershin and M. Di Ventra, Practical approach to programmable analog circuits with memristors, IEEE Transactions on Circuits and Systems; 2010, 57, p1857.
5. B. Muthuswamy, Implementing Memristor Based Chaotic Circuits, International Journal of Bifurcation and Chaos; 2010, 20, p1335.

6. B. Strukov, G.S. Snider, D.R. Stewart, and R.S. Williams, The missing memristor found, Nature 2008, 453, p80

7. B. Muthuswamy and L.O. Chua, simplest chaotic circuit, International Journal of Bifurcation and Chaos; 2010, 20, p1567.

8. B. Bao, T. Jiang, G. Wang, P. Jin, H. Bao, and M. Chen, An Improved Memristive Diode Bridge-Based Band Pass Filter Chaotic Circuit, Nonlinear Dynamics; 2017, 89, p1157 